\title{
Valores, suporte psicossocial e impacto do treinamento no trabalho ${ }^{1}$
}

\author{
Maria Júlia Pantoja \\ Universidade Católica de Brasília \\ Juliana Barreiros Porto \\ Universidade Presbiteriana Mackenzie \\ Luciana Mourão \\ Fundação Getúlio Vargas \\ Jairo Eduardo Borges-Andrade \\ Universidade de Brasília
}

\begin{abstract}
Resumo
O presente trabalho teve como objetivo investigar valores individuais e percepções de suporte organizacional à transferência de treinamento como preditores do impacto do treinamento no trabalho. A amostra consistiu de 79 profissionais, de nível superior, de uma instituição hospitalar na área de reabilitação do aparelho locomotor e de 85 profissionais de uma autarquia federal ligada ao sistema financeiro nacional. Os pesquisados haviam concluído os cursos de capacitação que foram objeto de avaliação neste estudo, há aproximadamente três meses. Responderam, no local de trabalho, questionários que continham escalas previamente validadas. Foram realizadas análises de regressão múltipla hierárquica. Os resultados apontaram suporte psicossocial e o tipo motivacional conservadorismo/coletivismo como importantes preditores do impacto do treinamento no trabalho $\left(R^{2}=0,35 ; p<0,001\right)$ e sugerem que os indivíduos que valorizam a conservadorismo, ou seja, que buscam respeito, compromisso, segurança, harmonia e aceitação dos costumes e idéias de uma cultura perceberam maior impacto dos conhecimentos e habilidades adquiridos nos cursos em suas atividades profissionais. A interação entre valores individuais e suporte organizacional à transferência foi marginalmente significativa. No entanto, sugere-se a confirmação desses resultados, a partir do aumento da amostra e da diversificação das organizações estudadas.
\end{abstract}

Palavras-chave: valores humanos; suporte psicossocial; impacto de treinamento

\begin{abstract}
Values, psychosocial support and training impact at work. The purpose of this work was to investigate individual values and perceptions of the organizational support to training's transfer as predictors of the impact in work training. Seventy nine university graduated degree professionals, working at a hospital specialized in orthopedics' rehabilitation plus eighty five professionals, working for an state government institution, connected to the national financial system, constituted the sample of this research. All of them had finished the training event which was evaluated in this paper approximately three months ago. While at work they responded to the questionnaire, which had scales previously validated. Multiple regression hierarchical analyses were made. The data shows psychosocial support and the motivational type conservationism|collectivism as an important predictor of the impact of the training at work $\left(R^{2}=0.35 ; p<\right.$ 0.001). It also suggests that people who value conservationism, i.e., that look for respect, compromise, safety, harmony and welcoming of culture habits and ideas got more from the impact of the knowledge and abilities acquired from their professional activities. The interaction between individual values and organizational support transfer had poor significance. Nonetheless, the confirmation of these data suggests that further studies should be undertaken by expanding the sample and diversifying the institutions studied.
\end{abstract}

Keywords: human values; psychosocial support; training impact

A capacitação e o desenvolvimento de profissionais para atuarem de forma integrada e competente, em um cenário mundial globalizado, no qual são permanentemente lançados novos conhecimentos e tecnologias, constitui-se em instigante desafio no campo de Treinamento e Desenvolvimento (T\&D). Diante disso, a eficácia dos programas instrucionais emerge como uma das mais relevantes questões tanto para as organizações 
quanto para os indivíduos em suas diversas áreas de atuação profissional.

A conceituação de treinamento engloba três importantes dimensões. A primeira refere-se à intencionalidade na melhoria do desempenho profissional, a segunda diz respeito ao controle desse processo por parte da organização e a terceira enfoca sua natureza processual.

Treinamento é definido por Goldstein (1991) como a aquisição sistemática de atitudes, conceitos, conhecimentos e habilidades que resultam em uma melhoria do desempenho no trabalho. De acordo com Wexley (1984), o processo de treinamento caracteriza um esforço por parte da organização, com vistas a facilitar a aprendizagem de comportamentos funcionais. A definição proposta por Hinrichs (1976) envolve essas duas dimensões, e amplia o conceito de treinamento ao incluir todas as possíveis modalidades de habilidades e quaisquer procedimentos de iniciativa da organização.

Tem havido certa confusão na delimitação do conceito de treinamento. Muitas vezes os termos desenvolvimento, educação e treinamento têm sido utilizados equivocadamente como sinônimos. Para Nadler (1984), torna-se importante e necessária a distinção entre tais conceitos, pois se referem a três tipos muito distintos de atividades. Treinamento visa apenas, segundo o referido autor, melhorar o desempenho do empregado no cargo que ocupa. Educação refere-se às oportunidades dadas pela organização para que o empregado tenha seu potencial desenvolvido, por meio da aprendizagem de novas habilidades que o capacitem a ocupar novos cargos dentro da mesma organização. Desenvolvimento de pessoal, entretanto, é um conceito mais abrangente e refere-se ao conjunto de experiências e de oportunidades de aprendizagem proporcionadas pela organização e que aumenta a possibilidade de crescimento pessoal dos empregados.

Nessa perspectiva, a análise histórica e política do campo de $T \& D$ ressalta a dimensão conservadora do processo de treinamento nas organizações de trabalho. O treinamento como solução para problemas relativos a competência técnica, vem sendo utilizado para ajustar os indivíduos aos seus novos papéis na estrutura da empresa, tendo como funções primordiais a capacitação técnica dos membros da organização e a modelagem de tipos de comportamentos compatíveis com os valores e os interesses organizacionais.

O treinamento, ao atingir cargos mais altos da organização, diz respeito, sobretudo, aos valores, sendo propósito maior a transmissão do conjunto de idéias que sintetizam a filosofia, a política e as diretrizes das organizações contemporâneas. De fato, dados de pesquisa recente na área de avaliação de treinamento (Rodrigues, 2000) indicam a existência de relacionamento positivo entre comprometimento organizacional e impacto do treinamento no trabalho. Os resultados sugerem que indivíduos comprometidos com a filosofia e com os valores da organização tendem a apresentar níveis mais altos de transferência de novas aprendizagens no ambiente de trabalho.
Treinamento, sob o enfoque instrucional (BorgesAndrade, 1986), pode ser definido como o modo como os profissionais de educação e treinamento conhecem, compreendem e predizem as questões concernentes ao ensino e à aprendizagem, bem como às mudanças de desempenho que se desejam de um determinado indivíduo e o que se faz com o propósito de obter mudanças. O modo como esse enfoque é usado, para resolver problemas de treinamento, é denominado Tecnologia Instrucional. Sob esse ponto de vista, o treinamento pode ser compreendido como um sistema composto por elementos ou subsistemas que mantêm entre si e com o ambiente externo um estreito relacionamento de interdependência. Os elementos que o compõem são avaliação de necessidades, planejamento de treinamento e avaliação de treinamento, este último foco do presente estudo.

Esses componentes mantêm entre si um constante fluxo de informações e produtos, sendo que o subsistema avaliação de treinamento é o principal responsável pelo fornecimento de informações para aperfeiçoamento constante do subsistema treinamento (Borges-Andrade, 2002).

Várias pesquisas brasileiras têm buscado encontrar preditores para o impacto do treinamento no nível do comportamento no cargo. Os preditores desse tipo de resultado vêm sendo focalizados em várias pesquisas brasileiras (Abbad, 1999; Carvalho, 2003; Lacerda, 2002; Lima, BorgesAndrade, \& Vieira, 1989; Meneses, 2002; Pantoja, 1999; Sallorenzo, 2000; Tamayo, 2002; Zerbini, 2003). Os resultados encontrados apontam as variáveis de apoio psicossocial à transferência, referentes ao contexto pós-treinamento, como determinantes da aplicação eficaz de desempenhos aprendidos em treinamento no ambiente de trabalho.

Aliados a isso, resultados de estudos empíricos realizados recentemente no campo de T\&D e apresentados por Sallas e Cannon-Bowers (2001) vêm indicando a importância de fatores pessoais (cognitivos, afetivos e motivacionais) na eficácia do treinamento. Entre esses fatores pessoais encontram-se variáveis como locus de controle, auto-eficácia, estilos de aprendizagem, motivação para aprender, hábitos de estudo e autoconceito profissional.

O presente trabalho descreve um estudo conduzido em duas instituições, uma na área de saúde, e outra relacionada ao sistema financeiro nacional. $\mathrm{O}$ objetivo foi o de investigar a influência de variáveis relacionadas às características dos treinados, mais especificamente, seus valores individuais, bem como de suas percepções acerca do suporte organizacional à transferência de treinamento, sobre tal impacto.

$\mathrm{O}$ texto encontra-se estruturado em três seções. Na primeira, são enfocadas questões teórico-conceituais relacionadas aos temas avaliação de treinamento, suporte organizacional à transferência e valores individuais. Na segunda, especificam-se procedimentos metodológicos utilizados na pesquisa. Na terceira seção, são analisados e discutidos os principais resultados encontrados. 


\section{Avaliação de treinamento e características organizacionais e individuais}

\section{O subsistema avaliação de treinamento}

Nas organizações, a área de treinamento e desenvolvimento tem cada vez mais sido reconhecida como estratégica. Mas, quais os resultados que ela realmente produz? Na fase de avaliação de treinamento, uma questão básica a ser respondida é se de alguma maneira ou sob algum critério o curso “valeu a pena”. Salas e Cannon-Bowers (2001) analisam que, hoje, há uma preocupação crescente nas organizações de que o investimento em treinamento seja justificado em termos de melhoria organizacional, de aumento da produtividade ou do desempenho.

Dentro dessa perspectiva, o modelo de análise de resultados de treinamento desenvolvido por Kirkpatrick (1976), posteriormente ampliado por Hamblin (1978), especifica os diversos tipos de efeitos dos programas instrucionais nas organizações. São eles: reações, aprendizagem, comportamento no cargo ou impacto do treinamento, organização e valor final. O nível de avaliação denominado comportamento no cargo focaliza o impacto do treinamento sobre o desempenho do profissional treinado em seu contexto de trabalho e se constitui no objeto do presente trabalho. Quanto à avaliação de treinamento no nível organizacional, o que se deseja efetivamente medir são mudanças que o treinamento possa ter provocado na organização. O foco deve ser no comportamento da organização ou de suas unidades. Já a avaliação do impacto no nível do "valor final” refere-se aos resultados, em geral com utilização de indicadores e de procedimentos pertencentes ao campo das ciências econômicas, estando fundamentados em cálculos de retorno sobre investimento. Hamblin (1978) avaliava que esses cinco níveis mantinham entre si um forte relacionamento positivo. Mas outros autores, como Alliger e Janak (1989) e Abbad (1999), têm mostrado que esses relacionamentos nem sempre são significativos ou nem sequer positivos. Abbad (1999) mostra que "resultados de algumas pesquisas têm mostrado situações em que um participante, apesar de demonstrar satisfação (reação favorável) com o treinamento e de ter obtido bons escores nas avaliações de aprendizagem, não aplicava, no trabalho, as novas habilidades que aprendera” (p. 36). Contudo, Salas e Cannon-Bowers (2001) confirmam que, apesar dos questionamentos em relação ao encadeamento positivo entre reações e saber, a tipologia de Kirkpatrick (1976) continua sendo a estrutura mais popular para guiar avaliações. Assim, o impacto do treinamento no trabalho corresponde ao terceiro nível de resultado de treinamento e focaliza os efeitos do treinamento sobre o desempenho apresentado pelo treinando quando do seu retorno ao contexto de trabalho, não sendo tal impacto necessariamente explicado pelos resultados de reação ao curso ou de aprendizagem. Nesse nível, busca-se verificar em que medida as habilidades e os conhecimentos adquiridos no treinamento são mobilizados, integrados e aplicados pelo treinado, no desenvolvimento de suas atividades profissionais.
Dados provenientes de pesquisas (Abbad, 1999; Pantoja, 1999; Sallorenzo, 2000) envolvendo a influência de variáveis relacionadas ao contexto organizacional sobre medidas de impacto de treinamento evidenciaram o importante papel das condições psicossociais na predição da eficácia dos treinamentos e sugerem a necessidade de que profissionais treinados, quando do seu retorno dos cursos, encontrem um clima/ suporte favorável à transferência dos novos conhecimentos.

\section{Suporte organizacional à transferência de treinamento}

O reconhecimento de que clima/suporte organizacional à transferência de treinamento é um fator crítico no estudo das variáveis que afetam a eficácia de programas instrucionais tem norteado a realização de pesquisas no campo de treinamento e desenvolvimento. Nesses estudos, a definição de clima/suporte à transferência envolve variáveis presentes no ambiente organizacional antes, durante e depois do evento instrucional. As variáveis que são incluídas geralmente são suporte gerencial à transferência, apoio e receptividade do grupo de trabalho à aplicação das novas aprendizagens, “dicas” situacionais (relativas aos objetivos, desenho da tarefa, autocontrole para a utilização do aprendido), bem como conseqüências (feedback, feedback negativo, punição e nenhum feedback) diante das tentativas do treinado de aplicar no trabalho as novas habilidades (Broad, 1982; Lima et al., 1989; Roullier \& Goldstein, 1993).

Alguns estudos voltados ao desenvolvimento de escalas para medir clima para transferência proporcionaram avanços teóricos e metodológicos significativos na área de avaliação de impacto de treinamento no trabalho. No estudo de Roullier e Goldstein (1993), por exemplo, que resultou na construção e validação de uma escala para medir o clima de apoio ao comportamentos aprendidos no treinamento, as variáveis relacionadas ao apoio psicossocial à transferência foram responsáveis pela maior parte da explicação do aproveitamento dos cursos no trabalho.

Parcialmente influenciadas por esse estudo, Abbad, Sallorenzo e Gama (1998) validaram uma escala de suporte organizacional à transferência de treinamento, utilizada no presente estudo, visando verificar o apoio gerencial, social, material e financeiro fornecido pelo contexto de trabalho à transferência das novas aprendizagens.

Segundo Abbad e Sallorenzo (2001), há fortes evidências empíricas mostrando, por exemplo, que uma nova habilidade aprendida em treinamento, muitas vezes não é transferida para o trabalho por falta de apoio organizacional. Inúmeros exemplos da literatura estrangeira e nacional sobre avaliação de treinamento demonstram que variáveis do contexto organizacional são importantes preditoras de impacto do treinamento no trabalho (Alves \& Tamayo, 1993; BorgesAndrade, 1982; Borges-Andrade \& Abbad, 1996; Broad, 1982; Campbell, 1988; Eisenberger, Huntington, Hutchinson, \& Sowa, 1986; Lima \& Borges-Andrade, 1985; Peters \& O’Connor, 1980; Roullier \& Goldstein, 1993).

A despeito da extrema importância de fatores contextuais à transferência positiva de treinamento, resultados de pes- 
quisas recentemente analisados e apresentados por Salas e Cannon-Bowers (2001) indicam que, na última década, houve uma proliferação de estudos sobre a influência das características pessoais do treinando. Algumas das variáveis que aparecem nas pesquisas encontradas pelos autores são: autoeficácia, orientação para objetivos, expectativas, motivação para aprender, sobre resultados de eventos instrucionais. Os resultados das pesquisas sobre essas variáveis apontam que elas constituem boas preditoras de eficácia do treinamento, tal como mensurada em termos de aprendizagem, reação e transferência (Cole \& Latham, 1997; Colquitt, Le Pine, \& Noe, 2000; Warr \& Bunce, 1995).

O construto auto-eficácia ocupa posição de destaque na revisão de literatura conduzida por Sallas e Cannon-Bowers (2001). O conceito de auto-eficácia foi desenvolvido por Bandura (1977b) no contexto da Teoria do Aprendizado Social. Auto-eficácia é a crença sobre a habilidade pessoal de desempenhar com sucesso determinadas tarefas ou comportamentos para produzir um resultado desejável (Bandura, 1977a). Segundo os autores, os dados das pesquisas analisadas fornecem evidências consistentes de que as crenças de auto-eficácia exercem influência positiva sobre a aprendizagem e desempenho dos treinandos (Ford, Smith, Weissbein, Gully, \& Salas, 1998; Phillips \& Gully, 1997; Stajkovic \& Luthans, 1998; Stevens \& Gist, 1997).

Orientação para objetivos é outra importante variável que tem recebido considerável atenção por parte de pesquisadores e teóricos do campo de treinamento e desenvolvimento (Brett \& VandeWalle, 1999; Phillips \& Gully, 1997). É amplamente conceituada como a estrutura mental utilizada pelo indivíduo para interpretar e se comportar em atividades orientadas para aprendizagem ou realização. Duas classes de orientação foram identificadas orientação para objetivos de aprendizagem e orientação para objetivos de desempenho. De acordo com Salas e Cannon-Bowers (2001), resultados de estudos recentes têm indicado que, em geral, a orientação para objetivos exerce influência sobre a transferência de treinamento, bem como está positivamente relacionada a autoeficácia (Ford et al., 1998; Phillips \& Gully, 1997).

Vários estudos têm fornecido evidências corroborando a concepção de que a motivação do treinando para aprender apresenta efeitos sobre a aquisição, retenção e aplicação dos conhecimentos e habilidades adquiridos em treinamento. Entre eles destaca-se o estudo de meta-análise conduzido por Colquitt et al. (2000), com importantes implicações para a pesquisa e a prática no campo de treinamento e desenvolvimento. De fato, os autores ressaltam a necessidade de expandir os tipos de variáveis de personalidade examinadas mais recentemente para incluir as emoções e a adaptabilidade, entre outras, bem como para verificar seus efeitos sobre a eficácia do treinamento.

Na literatura sobre aprendizagem, observa-se que a maioria das pesquisas na linha que investiga os efeitos da personalidade e da motivação sobre a aprendizagem se concentra nos fatores de motivação acadêmica: motivação para a reali- zação, locus de controle e autoconceito. Ros, Grad e Martinez (1996) apontam que as pesquisas têm focado apenas na motivação para a realização e não têm abordado a relação entre os processos de aprendizagem e as prioridades valorativas dos estudantes.

Em consonância com essa perspectiva, cabe ressaltar a agenda de pesquisa na área de treinamento e desenvolvimento proposta por Borges-Andrade e Abbad (1996). No que se refere especificamente à área de características da clientela, os autores recomendam que sejam investigadas variáveis como a auto-eficácia, a motivação para aprender, o locus de controle, bem como outras advindas da Psicologia Social.

Diante disso, estudos que focalizem o relacionamento entre os valores individuais dos treinandos, suporte à transferência e impacto de treinamento parecem úteis e relevantes para que maiores avanços teóricos possam ocorrer na área de avaliação de treinamento.

\section{Valores individuais}

A partir de um estudo de três requisitos básicos à existência humana, dos seus fundamentos na Filosofia, Sociologia e Psicologia e dos estudos de Rokeach (1973), Schwartz (1992) propõe uma teoria unificadora para o campo de motivação humana a fim de organizar as diferentes necessidades, motivos e objetivos propostos em outras teorias. Segundo Schwartz (2005b), os valores são caracterizados pelos seguintes aspectos: (1) valores são crenças intrinsecamente ligadas à emoção; (2) valores são um construto motivacional, pois se referem a objetivos desejáveis que as pessoas despendem altos níveis de esforços para alcançar; (3) valores transcendem situações e ações específicas; (4) valores servem como critérios para guiar a seleção e avaliação de ações, políticas, pessoas e eventos; e, (5) os valores são ordenados pela importância relativa aos demais. Assim, valores são definidos como critérios que transcendem situações específicas, que são ordenados por sua importância e que servem como princípios que guiam a vida do indivíduo (Schwartz, 1999).

A relação entre valores e comportamento tem sido um dos principais objetivos de pesquisa na área de valores (Schwartz, 1996). Desde Rokeach (1973), pesquisadores têm investigado a predição de um único valor ou uma lista de valores com o comportamento alvo. O desenvolvimento de teorias recentes, como o modelo de Schwartz (Schwartz, 1992; Schwartz \& Bilsky, 1987; Schwartz \& Bilsky, 1990), tem possibilitado avanços nesse estudo e constitui-se um campo fértil para os pesquisadores. Pesquisas têm demonstrado a relação entre valores e comportamentos (De Dreu \& Van Lange, 1995; Porto, 1998; Ros, 2001; Sagiv \& Schwartz, 1995; Schwartz, 1996; Schwartz \& Huismans, 1995), indicando a importância dessa variável para a compreensão dos comportamentos. Schwartz (1996) ressalta que a relação entre essas variáveis pode ser reforçada quando os comportamentos estudados implicam em conflito de valores, pois nessas situações os valores são ativados e são utilizados como princípios que guiam a vida do indivíduo. 
Em 1987, Schwartz e Bilsky derivaram uma tipologia universal de valores baseada na literatura sobre as necessidades básicas dos indivíduos. Em 1992, a teoria foi reformulada e foram identificados 56 valores classificados em 10 tipos motivacionais universais (Schwartz, 1992): poder (busca e preservação de uma posição social dentro de um sistema social); auto-realização (sucesso pessoal através da demonstração de competência de acordo com as regras sociais); hedonismo (prazer e gratificação para si mesmo); estimulação (novidade e estimulação na vida); autodeterminação (independência de pensamento e ação); filantropia (compreensão e proteção do bem-estar de todos e da natureza); benevolência (preocupação com o bem-estar de pessoas próximas); tradição (respeito, compromisso e aceitação dos costumes e idéias de uma cultura ou religião); conformismo (restrição de ações e impulsos que podem magoar outros ou violar as expectativas e as normas sociais); segurança (segurança, harmonia e estabilidade da sociedade, dos relacionamentos e do self).

O modelo teórico de Schwartz (1992) também prevê uma estrutura dinâmica entre os tipos motivacionais, de forma que as pessoas tendem a apresentar alta ou baixa prioridade para certos tipos motivacionais mutuamente compatíveis com certos domínios motivacionais do que de forma randômica. Em contrapartida, a priorização simultânea de alguns domínios incompatíveis levaria a conflitos.

Esses 10 tipos motivacionais podem ser classificados em tipos motivacionais de segunda ordem, em que poder e auto-realização são agrupados em autopromoção; hedonismo, estimulação e autodeterminação indicam abertura à mudança ou individualismo; filantropia e benevolência se enquadram na autotranscendência; e tradição, conformismo e segurança formam o tipo conservadorismo ou coletivismo. Esses tipos motivacionais de segunda ordem representam as compatibilidades dentro de cada agrupamento e os conflitos entre eles, ou seja, abertura à mudança versus conservadorismo e autopromoção versus autotranscendência (Schwartz, 1992).

Para avaliar empiricamente a teoria, foi desenvolvido o Inventário de Valores de Schwartz, que foi validado em 67 países. Os resultados deram apoio para a teoria e a existência de uma estrutura próxima ao universal. Além disso, a teoria foi testada utilizando-se outros métodos de medida e várias formas de análise de dados e os resultados foram consistentes com a teoria (Schwartz, 2005a; 2005b). Além da validação da estrutura dos valores, Schwartz buscou investigar a aplicabilidade da teoria de valores para predizer comportamentos. Schwartz (2005a) apresenta uma série de evidências empíricas sobre a relação entre valores e comportamentos de cooperação, votação e compra de produtos ecologicamente corretos.

A relação entre valores e comportamento é explicitada por Sagiv e Schwartz (1995). Esses autores apontam que as prioridades axiológicas podem dividir a atenção do indivíduo entre as oportunidades nas quais ele poderá atingir/alcançar metas particulares. Assim, as pessoas podem perceber situações como ocasiões para desempenhar ações relevantes para sua hierarquia de valores. Tipicamente os comportamentos não são guiados por um único comportamento, mas pela análise de compatibilidade e incompatibilidade entre valores antagônicos. Os dados empíricos dão suporte a relação entre comportamento e valores, mas as pressões normativas podem diminuir a expressão dos valores em comportamento (Schwartz, 2005a).

No ambiente organizacional, o desempenho das competências adquiridas no treinamento pode ser considerado como uma situação propícia para atingir determinadas metas; dessa forma os valores pessoais motivariam a aplicação de conhecimentos, habilidades e atitudes adquiridos no treinamento. Na medida em que os valores os impulsionam a agir e que o que foi aprendido é compatível com os valores dos participantes, seria possível observar uma relação entre os valores pessoais e a aplicação das competências no trabalho. Caso os valores não sejam compatíveis com os novos comportamentos esperados, pode ser interessante incluir no treinamento estratégias para modificar a prioridade de valores e tornar o treinamento mais eficaz. Em situação educacional, Ros (2001) relata o uso bem sucedido de estratégias de mudança de valores propostas por Rokeach (1973) para aprimorar o rendimento acadêmico, o que poderia ser aplicado também para a situação de treinamento.

Na área educacional, Ros, Grad e Martinez (1996) investigaram a relação entre rendimento e satisfação acadêmicos com valores, autoconceito, hábitos de estudo e estratégias de aprendizagem dos alunos. Rendimento acadêmico está negativamente associado à estimulação e positivamente aos valores de segurança e auto-realização. Os autores concluíram que apenas esses valores têm relações fortes e diretas com rendimento e satisfação acadêmicos. Entretanto, valores parecem estar relacionados com os determinantes mais relevantes de rendimento acadêmico como autoconceito, autoestima e com os tipos de enfoque de aprendizagem.

No presente trabalho, a suposição básica é de que os valores individuais podem predispor os participantes de eventos instrucionais a desempenharem comportamentos e habilidades aprendidos em treinamentos que sejam coerentes com a sua hierarquia de valores, além de influenciarem a percepção do contexto organizacional.

Com base na literatura apresentada, foram definidas três hipóteses gerais:

H1: Os valores individuais dos treinados influenciam o impacto do treinamento no trabalho.

H2: As percepções dos treinados quanto ao suporte à transferência de treinamento fornecido pela instituição influenciam o impacto de treinamento no trabalho.

H3: Valores individuais dos treinados constituem-se como variável moderadora da relação entre percepções de suporte à transferência fornecido pela organização e impacto de treinamento no trabalho. 


\section{Método}

\section{Caracterização das organizações estudadas}

Foram duas as organizações-alvo desta pesquisa. A primeira constitui-se em instituição de serviço social autônomo, de direito privado e sem fins lucrativos. Sua missão consiste em prestar assistência médico-hospitalar qualificada e gratuita a todos os níveis da população e desenvolver atividades educacionais e de pesquisa no campo da saúde. Atualmente, essa organização é composta por unidades localizadas em Brasília, São Luiz, Salvador e Belo Horizonte.

A segunda organização pesquisada é uma autarquia federal do sistema financeiro nacional. A missão da instituição é ser o agente da sociedade para assegurar o equilíbrio monetário da economia e, dessa forma, oferecer as condições básicas para o crescimento a longo prazo. A organização está presente em dez capitais brasileiras: Belém, Belo Horizonte, Curitiba, Fortaleza, Porto Alegre, Rio de Janeiro, Recife, Salvador, São Paulo e Brasília. Nesta última capital, localiza-se a sede da organização.

\section{Caracterização dos cursos}

$\mathrm{Na}$ organização hospitalar, os cursos avaliados foram realizados durante a terceira fase do processo seletivo treinamento para os cargos de médico, enfermeiro e terapeuta funcional. Nessa fase, caracterizada pela participação ativa do treinando em situações reais de trabalho, são desenvolvidos programas de treinamento que se constituem em atividades de ensino-aprendizagem - módulos teóricos e práticos. Os cursos apresentaram carga horária média de 16 horas e abrangem as áreas de anatomia, neuroanatomia, enfermagem geral, goniometria, atividades de vida diária, estatística, informática, noções de radiologia, mapa e tópicos em neurologia.

Na autarquia federal, órgão do sistema financeiro nacional, os cursos apresentaram carga horária média de 20 horas e tratavam de conteúdos atitudinais, com desenvolvimento de habilidades gerenciais e pessoais com especial foco ao atendimento ao cliente.

\section{Procedimentos de amostragem}

A escolha dos participantes do estudo foi feita entre aqueles profissionais que concluíram o treinamento há aproximadamente três meses, nos cargos de médico, enfermeiro e terapeuta funcional - na organização hospitalar - e nos cargos de técnico, analista, assessor e coordenador - na organização ligada ao sistema financeiro nacional. O número de profissionais pesquisados na primeira organização foi de 79 e na segunda de 85 .

Com o objetivo de facilitar a recordação e a avaliação dos respondentes, uma vez que os mesmos podem ter realizado mais de um curso, foi informado quais cursos estariam sendo avaliados em ambas as organizações.

\section{Instrumentos de coleta de dados}

O questionário para avaliar impacto de treinamento no trabalho e suporte à transferência de treinamento utilizado no presente estudo foi construído e validado por Oliveira-Castro, Pilati e Borges-Andrade (1998) e revalidado em uma das organizações estudadas (Pantoja, 1999). O questionário era composto por 34 itens, divididos em duas partes. A primeira denominava-se Impacto de Treinamento no Trabalho, contendo 12 (doze) questões de auto-avaliação versando sobre: (a) oportunidades encontradas para aplicação dos novos conhecimentos; (b) diminuição de erros na execução de atividades relacionadas ao curso; (c) recordação dos conteúdos abordados no curso, (d) qualidade do trabalho em atividades relacionadas ou não ao curso; (e) motivação, autoconfiança e receptividade a mudanças após a realização do curso; e (f) freqüência de utilização das novas aprendizagens.

A segunda incluía 22 itens relativos ao Suporte à Transferência e subdividia-se em: (a) Fatores Situacionais de Apoio, contendo 9 itens elaborados para levantar dados sobre o apoio gerencial, social (do grupo de trabalho) e organizacional recebido para aplicação das habilidades adquiridas em treinamento, em termos de oportunidades para praticar novas habilidades, apoio fornecido pelo supervisor na identificação e solução de dificuldades relacionadas à transferência de aprendizagem, repasse de informações necessárias à aplicação dos novos conhecimentos e outros itens similares; (b) Suporte Material, que englobava 6 itens relacionados a qualidade, quantidade e disponibilidade de recursos materiais e financeiros, bem como à adequação do ambiente físico do local de trabalho à transferência de treinamento; e (c) Conseqüências Associadas ao Uso das Novas Habilidades, que continha 7 itens referentes às percepções dos profissionais treinados sobre elogios, manifestações de apoio, indiferença, entre outros, frente às tentativas para aplicação das novas habilidades no trabalho. O resultado da análise fatorial do instrumento constatou dois fatores: Suporte psicossocial ( $\alpha=0,91)$ e Suporte material $(\alpha=0,86)$, sendo que apenas o primeiro fator foi utilizado no presente trabalho.

Todos os itens deveriam ser avaliados por uma escala do tipo Likert. Para os 12 primeiros, utilizou-se uma escala de concordância que variava de 5 (concordo totalmente) a 1 (discordo totalmente). Para os itens 13 a 34, foi utilizada uma escala de freqüência que variava de 1 (nunca) a 5 (sempre).

Os valores individuais dos treinados foram investigados através da Escala de Valores Humanos de Schwartz (1992) traduzida e validada para o Brasil por Tamayo e Schwartz (1993), através do método da Smallest Space Analysis, SSA. O instrumento apresenta 60 valores que devem ser avaliados por uma escala de importância que varia de 0 a 6. Quanto maior o número, mais importante é o valor para a pessoa. Além disso, foram utilizados o -1 que representa valores opostos e o 7 que significa valor supremo. Os participantes deveriam ler toda a listagem e escolher, primeiramente, aquele de suprema importância; em seguida, aqueles opostos aos seus e, somente após isso, avaliar os valores utilizando a escala de 0 a 6 . 


\section{Procedimentos de análise de dados}

Os dados foram inicialmente submetidos a análises estatísticas exploratórias e descritivas para identificação de variáveis com distribuição não-normal, para a localização de outliers e para a análise do relacionamento existente entre as variáveis para verificação de multicolinearidade e singularidade entre as mesmas (Tabachnick \& Fidell, 2001).

As análises descritivas apontaram a existência de três casos extremos univariados e cinco multivariados, que foram eliminados do banco de dados. A amostra ficou composta por 153 casos. Não foi detectado mais que 1,3\% de casos omissos, portanto, optou-se por substituí-los pela média.

Os escores para os quatro tipos motivacionais foram padronizados para retirar o efeito de uso da escala, conforme aponta Hofstede (1984) e Leung e Bond (1989). Os escores de todas as variáveis foram, então, centralizados para neutralizar a multicolinearidade que ocorre quando se realizam análises com interações. Esse procedimento não altera o padrão de intercorrelação das variáveis (Tabachinick \& Fidell, 2001).

Para analisar as relações existentes entre as variáveis (valores individuais, suporte à transferência e a interação entre essas com a variável-critério: impacto de treinamento no trabalho) foi realizada análise de regressão múltipla hierárquica. O primeiro modelo foi constituído pelos tipos motivacionais de segunda ordem (autopromoção, autotranscendência, conservadorismo e abertura à mudança), no segundo foram incluídas as percepções de suporte psicossocial e material, a seguir, as interações entre valores individuais e suporte psicossocial e, por último, as interações entre valores e su- porte material. As interações foram criadas visando investigar o efeito moderador dos valores sobre a relação entre suporte e impacto do treinamento.

Após a realização da regressão, foi realizada uma equação estrutural visando confirmar o modelo encontrado.

\section{Resultados e Discussão}

O objetivo desse trabalho foi investigar o poder preditivo dos valores individuais e percepções de suporte psicossocial à transferência de treinamento como preditores do impacto do treinamento no trabalho, bem como, o efeito moderador dos valores individuais sobre a relação entre os suportes psicossocial e material e o impacto do treinamento.

Os resultados da análise de regressão hierárquica indicaram que o suporte à transferência e os valores individuais do treinado estão significativamente relacionadas ao impacto do treinamento no trabalho. Mais especificamente, os dados obtidos apontaram o suporte psicossocial e o tipo motivacional conservadorismo como importantes preditores do impacto do treinamento no trabalho $\left(R^{2}=0,35 ; p<0,001\right)$.

O primeiro modelo, somente com os valores individuais, explicou $8 \%$ da variância, sendo que os tipos motivacionais conservadorismo e autopromoção foram os que apresentaram contribuição significativa, porém após a inserção da variável suporte psicossocial, apenas conservadorismo contribuiu significativamente. $\mathrm{O}$ acréscimo das interações foi marginalmente significativo para a interação entre suporte psicossocial e autotranscendência. A Tabela 1 apresenta o resultado da análise de regressão hierárquica (a matriz de

Tabela 1

Coeficientes de regressão, correlação, $R, R^{2}, R^{2}$ ajustado para a regressão hierárquica

\begin{tabular}{|c|c|c|c|c|c|c|c|c|}
\hline \multirow{3}{*}{ Preditores } & \multicolumn{8}{|c|}{ Modelos } \\
\hline & \multicolumn{2}{|c|}{1} & \multicolumn{2}{|c|}{2} & \multicolumn{2}{|c|}{3} & \multicolumn{2}{|c|}{4} \\
\hline & $\mathrm{B}$ & $\beta$ & B & $\beta$ & $\mathrm{B}$ & $\beta$ & B & $\beta$ \\
\hline 1. Abertura à mudança &, 06 & ,10 & ,02 &, 04 & ,03 &, 04 & ,02 &, 03 \\
\hline 2. Conservadorismo &, $22 * *$ & ,38 &, $21 * *$ &, 36 &, $19 * *$ &, 33 &, $19 * *$ &, 33 \\
\hline 3. Autotranscendência &,- 06 &,- 10 &,- 09 &,- 16 &,- 06 &,- 11 &,- 06 &,- 11 \\
\hline 4. Autopromoção &,$- 14^{*}$ &,- 19 &,- 07 &,- 10 &,- 07 &,- 10 &,- 08 &,- 11 \\
\hline 1. Suporte psicossocial & & &, $37 * *$ &, 52 &, $38 * *$ &, 54 &, $39 * *$ &, 55 \\
\hline 2. Suporte material & & & 01 &, 02 & ,03 & 05 & 03 & 05 \\
\hline 3. Suporte psicossocial x Abertura à mudança & & & & &,- 10 &,- 11 &,- 11 &,- 12 \\
\hline Suporte psicossocial x Conservadorismo & & & & &,- 13 &,- 16 &,- 06 &,- 07 \\
\hline Suporte psicossocial x Autotranscendência & & & & &, $17^{*}$ &, 22 &, 14 &, 18 \\
\hline Suporte psicossocial x Autopromoção & & & & &,- 17 &,- 15 &,$- 19 *$ &,- 16 \\
\hline 4. Suporte material x Abertura à mudança & & & & & & & ,01 &, 01 \\
\hline Suporte material x Conservadorismo & & & & & & &,- 09 &,- 13 \\
\hline Suporte material x Autotranscendência & & & & & & & ,02 &, 04 \\
\hline Suporte material x Autopromoção & & & & & & &, 01 &, 01 \\
\hline$R$ & \multicolumn{2}{|c|}{$0,328 * *$} & \multicolumn{2}{|c|}{$0,615 * *$} & \multicolumn{2}{|c|}{$0,651^{*}$} & \multicolumn{2}{|c|}{0,657} \\
\hline$R^{2}$ & \multicolumn{2}{|c|}{0,107} & \multicolumn{2}{|c|}{0,378} & \multicolumn{2}{|c|}{0,424} & \multicolumn{2}{|c|}{0,432} \\
\hline$R^{2}$ ajustado & \multicolumn{2}{|c|}{0,083} & \multicolumn{2}{|c|}{0,353} & \multicolumn{2}{|c|}{0,384} & \multicolumn{2}{|c|}{0,375} \\
\hline
\end{tabular}

$* p<0,05 ; * * p<0,01$ 
intercorrelações entre variáveis e interações encontra-se no Apêndice).

A modelagem por equação estrutural realizada teve como variáveis antecedentes do impacto do treinamento conservadorismo e suporte à transferência de treinamento (variável composta por suporte material e psicossocial). Os índices de ajustamento do modelo foram: $G F I=1,000$; $C F I=$ 0,998; RMSEA < 0,001; $R M R=0,018$. Todos os coeficientes foram significativos e podem ser observados na Figura 1. Entretanto, é importante ressaltar que os índices de normalidade multivariada estão marginalmente acima do esperado, o que pode acarretar o inflacionamento dos índices de ajuste e níveis de significância.

Tais evidências corroboram a relação entre valores e comportamento e os achados de pesquisas anteriores (De Dreu \& Van Lange, 1995; Porto, 1998; Sagiv \& Schwartz, 1995; Schwartz, 1996; Schwartz \& Huismans, 1995), sugerindo que os valores se constituem em variáveis importantes para a compreensão dos comportamentos dos indivíduos. Mais especificamente, os dados obtidos nesta pesquisa fornecem apoio empírico à relevância dos valores para fundamentar ações mais duradouras de mudança do comportamento no cargo; mudanças tais como aquelas do nível de avaliação denominado impacto do treinamento no trabalho. Portanto, os valores deveriam ser incluídos no conjunto de características individuais a serem mais bem examinadas por pesquisadores no campo de treinamento e desenvolvimento. De fato, no estudo de meta-análise conduzido por Colquitt et al. (2000), bem como na agenda de pesquisa proposta por BorgesAndrade e Abbad (1996) para a pesquisa sobre impacto do treinamento no trabalho, os autores recomendam que sejam expandidas as variáveis relativas às características da clientela para incluir as emoções, a adaptabilidade e outras advindas da Psicologia Social.

Além disso, à semelhança de resultados encontrados em diversas pesquisas (Abbad, 1999; Lima et al., 1989; Pantoja, 1999; Pilati \& Borges-Andrade, 2000; Pilati, Borges-Andrade, \& Posamai, 1999; Sallorenzo, 2000), os dados obtidos confirmam o importante papel das condições psicossociais na predição da transferência positiva de treinamento. Além disso, os resultados ressaltam a importância de uma cultura organizacional receptiva ao processo de aprendizagem contínua, atenta para estimular a aquisição, a recuperação, a criação e o intercâmbio de novos conhecimentos, idéias e experiências profissionais.

Os valores relacionados a conservadorismo ou coletivismo evidenciam a motivação do indivíduo para buscar respeito, compromisso, segurança, harmonia e aceitação dos costumes e idéias de uma sociedade ou cultura. Os resultados apontaram que indivíduos que valorizam mais o coletivismo tendem a perceber que aplicam mais as habilidades aprendidas. Em contrapartida, os indivíduos que valorizam mais a busca de poder, de êxito pessoal e de prazer não vêem na aplicação do aprendido a possibilidade de alcançar essas metas, pelo contrário, essas pessoas percebem que aplicam menos o aprendido no ambiente de trabalho. Entretanto, essa última relação foi baixa e pouco significativa no primeiro modelo e deixou de ser significativa após a inserção das demais variáveis. Esses dados estão, em parte, de acordo com os encontrados por Ros, Grad e Martinez (1996), já que os valores de Conservadorismo se relacionam positivamente: tanto com o rendimento, quanto com o impacto do treinamento. De

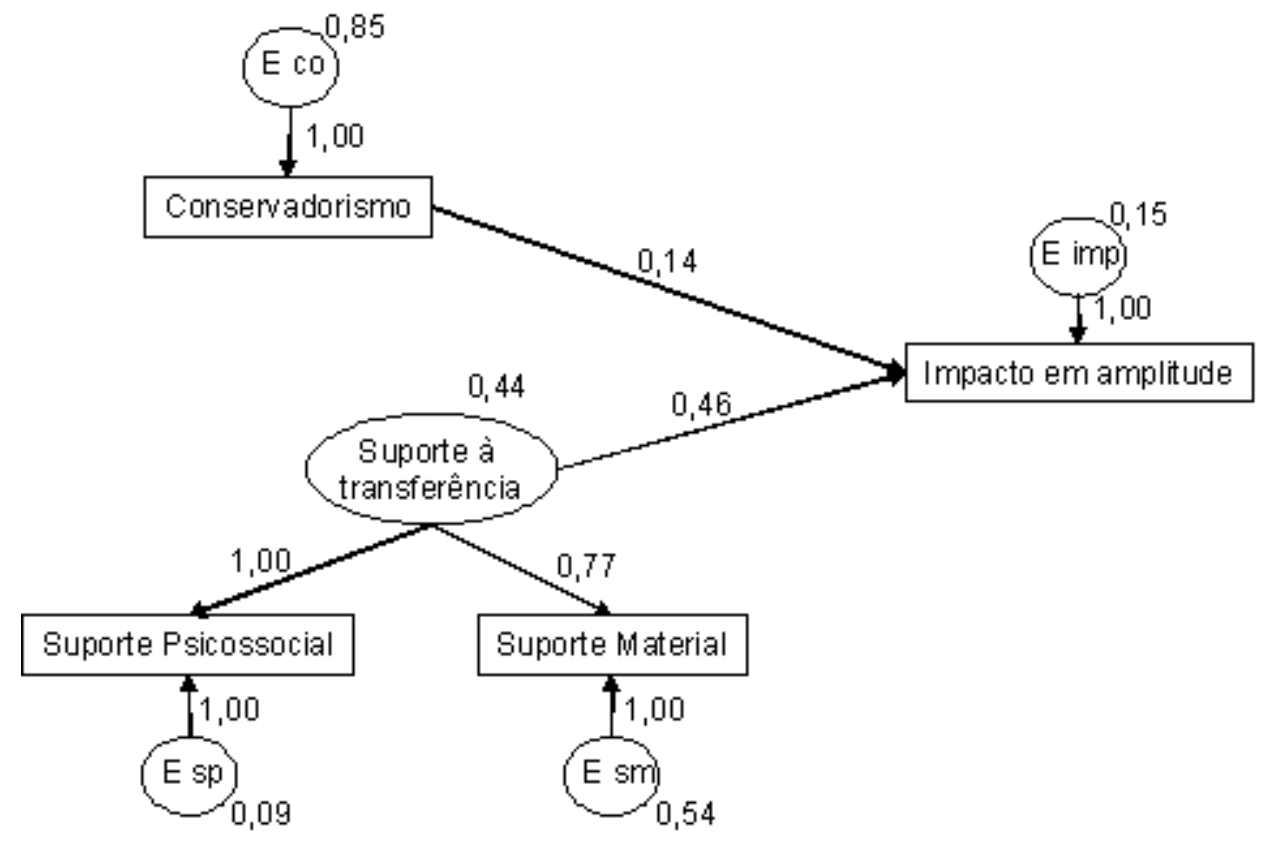

Figura 1. Equação estrutural para impacto do treinamento no trabalho. 
outro lado, a Auto-promoção, que engloba o tipo motivacional de auto-realização, não apresentou relação com o impacto do treinamento, ao passo que está associado ao rendimento acadêmico. Esses resultados podem estar sinalizando que os antecedentes de aprendizagem e de transferência de treinamento parecem apresentar distinções.

Cabe ressaltar que o poder explicativo dos valores foi pequeno, entretanto, os resultados estão de acordo com outros estudos que apontam para um efeito moderado dos valores sobre o comportamento (Schwartz, 2005a), em função da diversidade de fatores que influenciam o comportamento em ambientes naturais. Além disso, as pressões normativas para o desempenho em ambientes organizacionais é alta, o que pode diminuir significativamente a influência dos valores. Esse efeito também pode explicar a falta de relação negativa entre abertura à mudança e uso das competências adquiridas nos eventos instrucionais estudados, uma vez que teoricamente seria esperada essa relação em função da dinâmica de conflito entre tipos motivacionais. Já que a pressão para a execução do desempenho pode ser alta, o indivíduo com alta prioridade de abertura à mudança não tem a possibilidade de não aplicar o conhecimento. Assim, ele aplica os conhecimentos mesmo que isso cause conflito com seus valores. Entretanto, é possível que isso tenha conseqüências para outros comportamentos organizacionais, como o comprometimento organizacional, aspecto a ser explorado em pesquisas futuras.

Por definição, treinamento é uma ação organizacional planejada e sob controle com o objetivo de facilitar a aprendizagem de comportamentos funcionais. Os resultados sugerem que o treinamento tem cumprido o seu papel de manutenção do sistema organizacional e os indivíduos que valorizam a manutenção do status quo vêem, na aplicação das habilidades aprendidas, uma oportunidade para expressão de sua hierarquia de valores, pois a aplicação leva à manutenção de todo o sistema organizacional.

Esses resultados também estão de acordo com a pesquisa de Rodrigues (2000), que apresenta comprometimento organizacional como um importante preditor do uso de novos conhecimentos, habilidades e atitudes adquiridas em treinamento no trabalho, pois as pessoas que valorizam mais o conservadorismo tendem a apresentar maior comprometimento com a organização (Tamayo et al., 2001).

Finalizando, neste estudo, os resultados não permitem concluir que valores individuais se constituem como moderadores da relação entre suporte organizacional à transferência e o impacto do treinamento no trabalho. O efeito moderador foi pouco significativo e apenas a relação entre suporte psicossocial e autotranscendência foi significativa, indicando que as pessoas que valorizam mais autotranscendência, ou seja, que visam o bem-estar dos outros, apresentaram escores mais altos de impacto do treinamento no trabalho, do que as pessoas que valorizam menos o bem-estar comum. Como o suporte psicossocial, neste caso, está relacionado a apoiar as pessoas treinadas a aplicarem no trabalho o que aprenderam no treinamento, é possível concluir que a aplica- ção do que foi aprendido está associado à idéia de bem-estar comum. Isso confirma também a idéia central dos modelos de Kirkpatrick (1976) e Hamblin (1978) de que os resultados de treinamentos podem ir além do sujeito capacitado e provocar mudanças na organização, sendo, portanto, associados ao bem-estar comum.

É importante lembrar que o conceito de valores é distal ao de treinamento e, por isso, o seu efeito sobre as percepções de suporte e o comportamento de aplicar o aprendido é pequeno e, por conseguinte, amostras grandes são requeridas nessas situações para se obter resultados conclusivos, o que não foi o caso deste estudo. Talvez em função deste ponto, a relação moderadora não foi encontrada como previsto. Outro aspecto que pode ser responsável por esses resultados é o fato da percepção de suporte organizacional não envolver situações de conflito entre valores, assim essa percepção independe dos valores dos indivíduos sendo igualmente importante para todas as prioridades de valores.

Cabe ressaltar, entretanto, que dentro de um enfoque interacionista cuja suposição básica é de que características individuais interagem com fatores contextuais na explicação do comportamento humano no trabalho, é de suma importância que sejam mais intensamente investigadas as interações entre valores individuais e variáveis contextuais na explicação da eficácia da aprendizagem e da transferência positiva de aprendizagem no trabalho. Para tanto, é necessário confirmar esses resultados, a partir do aumento da amostra e da diversificação das organizações estudadas.

\section{Referências}

Abbad, G. (1999). Um modelo integrado de avaliação do impacto do treinamento no trabalho - IMPACT. Tese de doutorado não-publicada, Universidade de Brasília, Brasília.

Abbad, G., \& Sallorenzo, L. H. (2001). Desenvolvimento e validação de escalas de suporte à transferência de treinamento. Revista de Administração da Universidade de São Paulo - RAUSP, 36, 33-45.

Abbad, G., Sallorenzo, L., \& Gama, A. (1998). Percepção de suporte organizacional à transferência de treinamento: validação de um questionário [Resumo]. In Sociedade Brasileira de Psicologia (Org.), Resumos de comunicações científicas - XXVIII Reunião Anual de Psicologia (p. 205). Ribeirão Preto: Autor.

Alliger. G. M., \& Janak, E. A. (1989). Kirkpatrick’s levels of training criteria: thirty years later. Personnel Psychology, 42, 331-342.

Alves, A. R., \& Tamayo, A. (1993). Sistema de avaliação da Telebrás - SAT. Revista de Administração, 28, 73-80.

Bandura, A. (1977a). Self-efficacy: toward a unifying theory of behavioral change. Psychological Review, 84, 191-215.

Bandura, A. (1977b). Social Learning Theory. New Jersey: Prentice Hall.

Borges-Andrade, J. E. (1982). Avaliação somativa de sistemas instrucionais: integração de três propostas. Tecnologia Educacional, 11, 29-39.

Borges-Andrade, J. E. (1986). Por uma competência técnica no treinamento. Psicologia, Ciência e Profissão, 2, 9-17.

Borges-Andrade, J. E. (2002). Desenvolvimento de medidas em avaliação de treinamento. Estudos de Psicologia, 7(Número especial), 31-43.

Borges_Andrade, J. E., \& Abbad, G. (1996). Treinamento e desenvolvimento: reflexões sobre suas pesquisas científicas. Revista de Administração, 31, $112-125$. 
Brett, J. F., \& Vandevalle, D. (1999). Goal orientation and goal content as predictors of performance in a training program. Journal of Applied Psychology, $84,863-873$.

Broad, M. L. (1982). Management actions to support transfer of training. Training and Development Journal, 36, 124-130.

Campbell, J. P. (1988). Training design for performance improvement. In J. P. Campbell \& R. J. Campbell (Orgs.), Productivity in organizations (pp. 177-215). São Francisco: Josey Bass.

Carvalho, R. S. (2003). Avaliação de treinamento a distância via internet: reação, suporte à transferência e impacto do treinamento no trabalho. Dissertação de mestrado não-publicada, Universidade de Brasília, Brasília.

Cole, N. D., \& Latham, G. P. (1997). Effects of training in procedural justice on perceptions of disciplinary fairness by unionized employees and disciplinary subject matter experts. Journal of Applied Psychology, 82, 699-705.

Colquitt J. A, LePine J. A, \& Noe, R. N. (2000). Toward an integrative theory of training motivation: a metaanalytic path analysis of 20 years of research. Journal of Applied Psychology, 85, 678-707.

De Dreu, C. K. W., \& Van Lange, P. A. M. (1995). The impact of social values orientations on negotiator cognitions and behavior. Personality and Social Psychology Bulletin, 21, 1178-1188.

Eisenberger, R., Huntington, R., Hutchinson, S., \& Sowa, D. (1986). Perceived organizational support. Journal of Applied Psychology, 71, 500-507.

Ford, J. K., Smith, E. M., Weissbein, D. A., Gully, S. M., \& Salas, E. (1998). Relationships of goal-orientation, metacognitive activity, and practice strategies with learning outcomes and transfer. Journal of Applied Psychology, 83, 218-233.

Goldstein, I. L. (1991). Training in work organizations. In M. D. Dunnette \& L. Hough (Orgs.), Handbook of Industrial and Organizational Psychology (pp. 507-619). Palo Alto, California: Consulting Psychologists.

Hamblin, A. C. (1978). Avaliação e controle de treinamento. São Paulo: McGraw-Hill.

Hinrichs, J. R. (1976). Personnel training. In M. D. Dunnette (Orgs.), Handbook of Industrial and Organizational Psychology (pp. 1-27). Chicago: Rand McNally.

Hofstede, G. (1984). Culture's consequences, international differences in workrelated values. Newbury Park, California: Sage.

Kirkpatrick, D. L. (1976). Evaluation of training. In R. L. Craig (Org.), Training and development handbook (pp. 1-27). Nova York: Mc Graw-Hill.

Lacerda, E. R. M. (2002). Impacto do treinamento no trabalho: investigando variáveis motivacionais e organizacionais como suas preditoras. Dissertação de mestrado não-publicada, Universidade de Brasília, Brasília.

Leung, K., \& Bond, M. H. (1989). On the empirical identification of dimensions for cross-cultural comparisons. Journal of Cross-Cultural Psychology, 20, 133-151.

Lima, S. M. V., \& Borges-Andrade, J. E. (1985). Meta-análise de avaliação de treinamento, Revista de Administração, 20, 39-52.

Lima, S. M. V., Borges-Andrade, J. E., \& Vieira, S. B. A. (1989). Cursos de curta duração e desempenho em instituições de pesquisa agrícola. Revista de Administração, 24, 36-46.

Meneses, P. P. M. (2002). Auto-eficácia, locus de controle, suporte à transferência e impacto de treinamento no trabalho. Dissertação de mestrado nãopublicada, Universidade de Brasília, Brasília.

Nadler, L. (1984). The handbook oh human resources development. Nova York, Wiley.

Oliveira-Castro, G. A., Pilati, R., \& Borges-Andrade, J. E. (1998). Percepção de suporte organizacional: desenvolvimento e validação de um questionário [Texto completo]. In Associação Nacional dos Programas de Pós-graduação em Administração (Org.), Anais do $22^{\underline{0}}$ Encontro da ANPAD [CD-ROM]. Foz do Iguaçu: Autor.
Pantoja, M. J. (1999). Avaliação de impacto de treinamento na área de reabilitação. Preditores individuais e situacionais. Dissertação de mestrado nãopublicada, Universidade de Brasília, Brasília.

Peters, L. H., \& O'Connor, E. J. (1980). Situational constraints and work outcomes: the influence of frequently overlooked construct. Academy of Management Review, 82, 826-831.

Phillips, J. M., \& Gully, S. M. (1997). Role of goal orientation, ability, need for achievement, and locus of control in the self-efficacy and goal-setting process. Journal of Applied Psychology, 82, 792-802.

Pilati, R., \& Borges-Andrade, J. E. (2000). Impacto do treinamento em amplitude e profundidade: preditores individuais e situacionais [Resumo]. In Sociedade Brasileira de Psicologia (Org.), XXX Reunião da Sociedade Brasileira de Psicologia. Brasília: Autor.

Pilati, R., Borges-Andrade, J. E., \& Posamai, L. (1999). Impacto do treinamento em amplitude e profundidade: relações com suporte à transferência, gestão do desempenho e liberdade decisória. In Sociedade Brasileira de Psicologia (Org.), XXIX Reunião da Sociedade Brasileira de Psicologia (p. 53). Campinas: Autor.

Porto, J. B. (1998). Prioridades axiológicas e cultura brasileira como preditores de civismo nas organizações. Dissertação de mestrado não-publicada, Universidade de Brasília, Brasília.

Rodrigues, A. G. (2000). A natureza da participação e suas implicações no impacto do treinamento no trabalho. Dissertação de mestrado não-publicada, Universidade de Brasília, Brasília.

Rokeach, M. (1973). The nature of human values. Nova York: Free Press.

Ros, M. (2001). Valores, actitudes y comportamiento: una nueva visita a un tema clásico. In M. Ros, \& V. V. Gouveia (Org.), Psicología social de los valores humanos: desarrollos teóricos, metodológicos y aplicados (pp. 79-99). Madrid: Biblioteca Nueva.

Ros, M., Grad, H., \& Martinez, E. (1996). El cambio de valores para la mejora de las estratégias de aprendizaje y rendimiento acadêmico. Madrid: Informe CIDE. Mec.

Rouiller, J. Z., \& Goldstein, I. L. (1993). The relationship between organizational transfer climate and positive transfer of training. Human Resource Development Quarterly, 4, 377-390.

Sagiv, L., \& Schwartz, S. H. (1995). Value priorities and readiness for out-group social contact. Journal of Personality and Social Psychology, 69, 437-448.

Salas, E., \& Cannon-Bowers, J. (2001). The science of training: a decade of progress. Annual Review of Psychology, 52, 471-499.

Sallorenzo, L. H. (2000). Avaliação de impacto do treinamento no trabalho. Analisando e comparando modelos de predição. Dissertação de mestrado não-publicada, Universidade de Brasília, Brasília.

Schwartz, S. H. (1992). Universals in the content and structure of values: theoretical advances and empirical tests in 20 countries. In M. P. Zanna (Org.), Advances in experimental social psychology (v. 24, pp. 1-65). San Diego: Academic.

Schwartz, S. H. (1996). Value priorities and behavior: applying a theory of integrated value systems. In C. Seligman, J. M. Olson, \& M. P. Zanna (Orgs.), Psychology of values: the Ontario Symposium (v. 8, pp. 1-26). New Jersey: Lawrence Erlbaum.

Schwartz, S. H. (1999). A theory of cultural values and some implications for work. Applied Psychology: An International Review, 48, 23-47.

Schwartz, S. H. (2005a). Validade e aplicabilidade da teoria de valores. In A. Tamayo \& J. B. Porto (Orgs.), Valores e comportamento nas organizações. Rio de Janeiro: Vozes.

Schwartz, S. H. (2005b). Valores humanos básicos: seu contexto e estrutura intercultural. In A. Tamayo \& J. B. Porto (Orgs.), Valores e comportamento nas organizações. Rio de Janeiro: Vozes.

Schwartz, S. H., \& Bilsky, W. (1987). Toward a universal psychological structure of human values. Journal of Personality and Social Psychology, 53, 550-562. 
Schwartz, S. H., \& Bilsky, W. (1990). Toward a theory of the universal content and structure of values: extensions and cross-cultural replications. Journal of Personality and Social Psychology, 58, 878-891.

Schwartz, S. H., \& Huismans, S. (1995). Value priorities and religiosity in four western religions. Social Psychology Quarterly, 58, 88-107.

Stajkovic, A. D., \& Luthans, F. (1998). Self-efficacy and work-related performance. A metanalysis. Psychological Bulletin, 124, 240-261.

Stevens, C. K., \& Gist, M. E. (1997). Effects of self-efficacy and goal-orientation training on negotiation skill maintenance: what are the mechanisms? Personnel Psychology, 50, 955-978.

Tabachnick, B. G., \& Fidell, L. S. (2001). Using multivariate statistics (4⿳亠丷a Massachusets: Allyn \& Bacon.

Tamayo, A., \& Schwartz (1993). Estrutura motivacional dos valores. Psicologia: Teoria e Pesquisa, 9, 329-348.
Tamayo, A., Souza, M. G. S., Vilar, L. S., Ramos, J. L., Albernaz, J. V., \& Ferreira, N. P. (2001). Prioridades axiológicas e comprometimento organizacional. Psicologia: Teoria e Pesquisa, 17, 27-35.

Tamayo, N. (2002). Autoconceito profissional, suporte à transferência e impacto de treinamento no trabalho. Dissertação de mestrado não-publicada, Universidade de Brasília, Brasília.

Warr, P., \& Bunce, D. (1995). Trainee characteristics and the outcomes of open learning. Personnel Psychology, 48, 347-375.

Wexley, K. N. (1984). Personal training. Annual Review Psychology, 35, 519551.

Zerbini, T. (2003). Estratégias de aprendizagem, reações de um curso via internet, reações ao tutor e impacto do treinamento no trabalho. Dissertação de mestrado não-publicada, Universidade de Brasília, Brasília.

Maria Júlia Pantoja, doutora em Psicologia Organizacional e do Trabalho pela Universidade de Brasília, é professora dos cursos de Administração e mestrado em Gestão do Conhecimento da Universidade Católica de Brasília. Endereço para correspondência: Condomínio do Lago Sul, conjunto “C”, casa 5 (Lago Sul); Brasília, DF; CEP 71680-353. Tel: (61) 3367-5248 e (61) 9986-4703; Fax: (61) 3447-8000. E-mail: jpantoja@agricultura.gov.br Juliana Barreiros Porto, doutora em Psicologia Organizacional e do Trabalho pela Universidade de Brasília, é professora na pós-graduação da Universidade Presbiteriana Mackenzie e pesquisadora e consultora do Instituto de Ciências do Trabalho.E-mail: porto.juliana@gmail.com

Luciana Mourão Cerqueira e Silva, doutora em Psicologia Organizacional e do Trabalho pela Universidade de Brasília, é professora na pós-graduação da Fundação Getúlio Vargas e diretora de HOJE/EMP Consulting. E-mail: luciana.mourao@empconsulting.com.br

Jairo Eduardo Borges-Andrade, doutor em Sistemas Instrucionais pela Florida State University (Tallahassee, EUA), é professor titular no Departamento de Psicologia Social e do Trabalho, do Instituto de Psicologia, Universidade de Brasília. E-mail: jairo.borges@gmail.com 


\section{Apêndice}

Correlações entre as variáveis do estudo

\begin{tabular}{|c|c|c|c|c|c|c|c|c|c|c|c|c|c|c|}
\hline & $\begin{array}{l}\text { Impacto do } \\
\text { treinamento }\end{array}$ & 1 & 2 & 3 & 4 & 5 & 6 & 7 & 8 & 9 & 10 & 11 & 12 & 13 \\
\hline 1. Abertura à mudança & 01 & & & & & & & & & & & & & \\
\hline 2. Conservadorismo &, 28 & ,18 & & & & & & & & & & & & \\
\hline 3. Autotranscendência & ,15 &, 52 & ,71 & & & & & & & & & & & \\
\hline 4. Autopromoção &,- 08 &, 52 & ,26 & 35 & & & & & & & & & & \\
\hline 5. Suporte psicossocial &, 55 &, 07 & 09 &, 12 &,- 08 & & & & & & & & & \\
\hline 6. Suporte material &, 34 &, 12 & ,24 & ,32 &,- 12 &, 52 & & & & & & & & \\
\hline 7. Suporte psicossocial x Abertura à mudança &,- 07 & ,08 &, 10 & ,03 & ,07 &, 00 & 00 & & & & & & & \\
\hline 8. Suporte psicossocial x Conservadorismo & ,15 & ,08 & ,09 &, 13 &, 10 &, 28 & ,20 &,- 02 & & & & & & \\
\hline 9. Suporte psicossocial x Autotranscendência & ,16 & ,03 &, 12 &, 00 &, 10 & , 16 & 03 & ,33 & ,71 & & & & & \\
\hline 10. Suporte psicossocial x Autopromoção &,- 03 &, 07 &, 11 &, 12 &, 04 & ,20 &, 17 & ,44 &, 18 & ,22 & & & & \\
\hline 11. Suporte material x Abertura à mudança &,- 04 &,- 12 &,- 17 &,- 25 &,- 22 &,- 01 &,- 09 & ,45 &,- 11 &, 08 & 07 & & & \\
\hline 12. Suporte material x Conservadorismo &, 05 &,- 18 &,- 09 &,- 10 &,- 25 & ,20 &, 13 &,- 14 & ,44 & ,22 &,- 07 & ,16 & & \\
\hline 13. Suporte material x Autotranscendência &, 05 &,- 25 &,- 10 &,- 27 &,- 23 & ,02 &,- 11 & ,09 &, 23 & ,45 &,- 08 &, 50 & ,62 & \\
\hline 14. Suporte material x Autopromoção &, 01 &,- 21 &,- 23 &,- 21 &,- 41 &, 13 & ,22 &, 06 &,- 06 &,- 07 &, 22 & ,49 & ,28 & ,27 \\
\hline
\end{tabular}

